\title{
On Vibration Joint Time-Frequency Investigations of CNC Milling Machines for Tool Trajectory Task Conformity Estimation
}

\author{
Silviu Nastac \\ Research Centre for Mechanics of Machines and Technological Equipments, Engineering and Agronomy Faculty in Braila, \\ "Dunarea de Jos" University of Galati, Calea Calarasilor 29, Braila 810017, Romania \\ Correspondence should be addressed to Silviu Nastac; snastac@ugal.ro
}

Received 2 July 2018; Revised 14 August 2018; Accepted 6 September 2018; Published 10 October 2018

Academic Editor: Matteo Aureli

Copyright $(92018$ Silviu Nastac. This is an open access article distributed under the Creative Commons Attribution License, which permits unrestricted use, distribution, and reproduction in any medium, provided the original work is properly cited.

\begin{abstract}
This study deals with estimation of milling shape accuracy and trajectory conformity for small CNC 3D milling machines, based on vibration monitoring during the regular working cycles. The author made a large number of experimental tests, acquiring the acceleration signals, both on the milling tool-holder and on the bed frame. In order to evaluate the appropriate spectral characteristics of different machine parts and their weights on equipment dynamics, it was analyzed both the complete and the partial working cycle (such as forward tool motion, with or without effective milling, with or without tool driving, exclusively the milling cutter transitory/stabilized regime) for different basic milling shapes. The acceleration signals were jointly time-frequency investigated in order to evaluate specific spectral indicators related to the real motion characteristic of milling tool. It was used short time fast Fourier transform (STFFT) and Hough transform (HT) algorithms, along with stochastic evaluation of signal parameters, within time and frequency domains. The results reveal an accurate correlation between the specific transitory dynamics of the machine and the imposed milling shape. Main implications of the results within this analysis involve the noninvasive and facile investigation for milling errors of the $\mathrm{CNC}$ machine, conformity of tool head trajectory, identification of potential failure source, or damaged machine part.
\end{abstract}

\section{Introduction}

Nowadays, CNC milling machine, stands for computer numerical control, denotes a milling machine commonly used in manufacturing process for industrial fields. CNC machine performs the operations of drilling and turning and has various types of cutting tools used to cut wood, metal, or other materials. Each process of the CNC milling machine has to be considered a set of complex dynamic interactions between the machine tool mechanical structure and the workpiece cutting $[1,2]$. Izelu et al. [3] showed that the vibrations generated during CNC operations have multispectral composition and, in addition, depend directly by the manufacturing process, being a decisive factor in high-speed limiting of the processing velocity. Choudhury et al. [4] developed a system for online vibration control on a lathe, through correlation between cutting parameters. Dimla [5] described a tool-wear monitoring procedure in a metal turning operation, intended to correlate vibration signals with the tool wear. Particular attention was paid to the manner that the sensor signals acquired from the cutting process have been harnessed and used into the development of tool condition monitoring systems. Some researchers focus their study on characterization of the dynamic properties of a machine tool, during machining within the chosen revolution speed range. The natural frequency of each mode shape was estimated through the linear modal analysis approach, using finite element method or experimental/operational modal analysis, by the help of modal impact hammer testing specific procedures [6,7]. Patoommakesorna et al. [8] proposed a new algorithm (named Hough line transform) for a straight line matching, by integration of vision-based image processing. Using the same method, Robles et al. [9] obtained the effective localisation of cutting edges within the milling-head edge profile. Wu et al. [10] identified dynamic characteristics and developed fault diagnosis method of a CNC machine, by using a spectrum analysis procedure of the milling-tool vibration signals. In addition, Zaghbany and Songmene [11] investigated the operational modal analysis (OMA) for dynamic modal parameter estimations, 


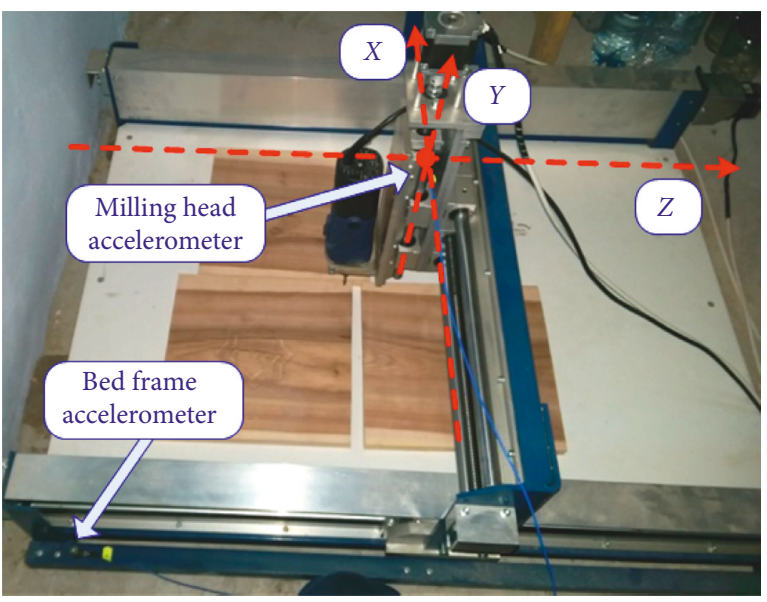

(a)

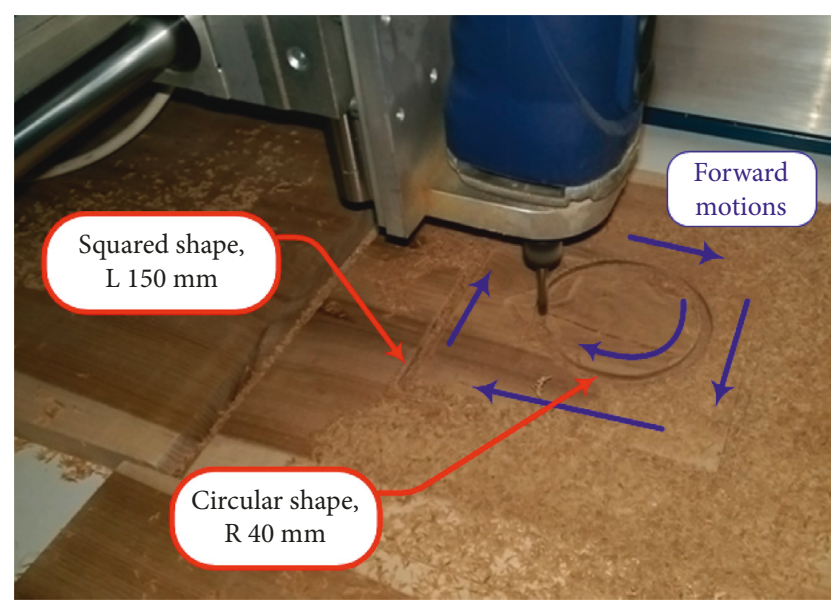

(b)

FIGURE 1: The general view of the experimental setup (a) with indication of measurement points and vibration monitoring directions and, respectively, a detailed view of the milling area (b) containing examples of milling shapes.

during machining operations. Bisu et al. [12] proposed a method for vibration analysis, in order to monitor the milling process quality online. The vibration envelope analysis was proposed to detect the cutting capacity of the tool, with application into the optimization of cutting parameters. Within her study [13], Debeleac proposed an analysis based on multibody model approach for mechanical systems with complex configuration and used an advanced computational method to investigate nonlinear dynamics of such systems. Gelman [14] proposed new higher order spectral technique, especially the cross covariance of complex spectral components, in order to monitor the damage level of structures and machineries.

Taking into account the large-scale utilization of $\mathrm{CNC}$ milling machines, the previously in-brief presentation of the actual studies, regarding dynamical aspects within exploitation of such machineries, reveals the high importance of development of new methods, which combine operational and computational investigations, in order to optimize technological capabilities of these milling equipment. In addition, the analyzed references dignify the opportunity and the particular contribution of advanced signal processing methods for the analysis and the characterization of CNC machine dynamics. Hereby, the significance of this study arises both from the operational noninvasive dynamic testing procedure (without any involvement on regular configuration of technological cycle) and from the proposed computational technique, based on a suitable combination between the joint time-frequency analysis and the HT algorithm for graphical shape identification. Obviously, the conformity of the tool trajectory task denotes the final desideratum of all studies, apart from its particular objective, which finally increases the milling process quality.

\section{Experimental Setup and Investigation Methods}

The images in Figure 1 present a general view of the experimental setup (a) and a detailed view of the effective milling area (b). On the picture in Figure 1(a) were marked the positions of the two acceleration sensors, with the motion-monitoring axis. In addition, on the image in Figure 1(b) were depicted both the two basic shapes that were analyzed during the milling process and the forward motions of the tool head. As it can seen in Figure 1, it was used a small CNC 3D milling machine working on wood samples (as experimental work pieces). During the experiments, it was supposed two different shapes as follows: a squared $150 \times 150 \mathrm{~mm}$ shape and, respectively, a circular shape with $40 \mathrm{~mm}$ radius. Both tasks were positioned in the same area of the wood sample, thus the run of the milling head, from the rest position, to the start position of the current task, approximately involves the same motions. Within the same hypothesis, the forward motions were set up onto the same direction.

Acceleration signals were acquired using a triaxial accelerometer, mounted on the milling tool-holder and a simple accelerometer mounted on the bed frame, near the milling area (Figure 1(a)). Acquisition setup also involves the hardware NI USB-9233 quad inputs DAQ board and a software application developed within NI-LabVIEW. Subsequent data postprocessing and analyzing were performed with some applications developed within the MATLAB software.

The experiments scheduler supposes four cases, applied for each milling shape, as follows: (i) milling-head running on the programmed shape trajectory without tool driving, (ii) idem first case, but with tool driving at regular speed and no interaction between milling cutter and workpiece, and (iii) the effective milling of the programmed shape. In addition, it was considered a supplementary case of no motion of the milling equipment (along $X, Y$, or $Z$ axes), just only driving the milling cutting tool (from start, through stabilized speed regime, to stop).

The first category of investigation methods contains frequency domain analyses, as follows: (a) the spectral composition of signals, with detection of significant 

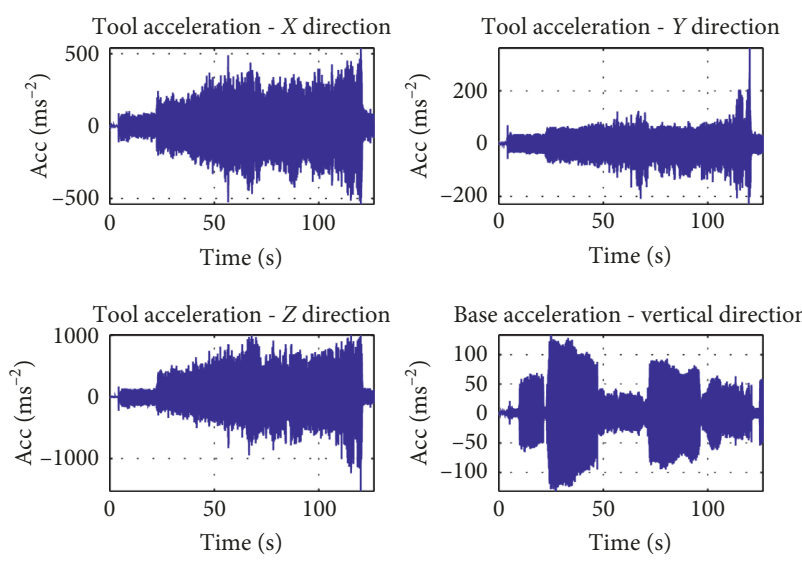

(a)
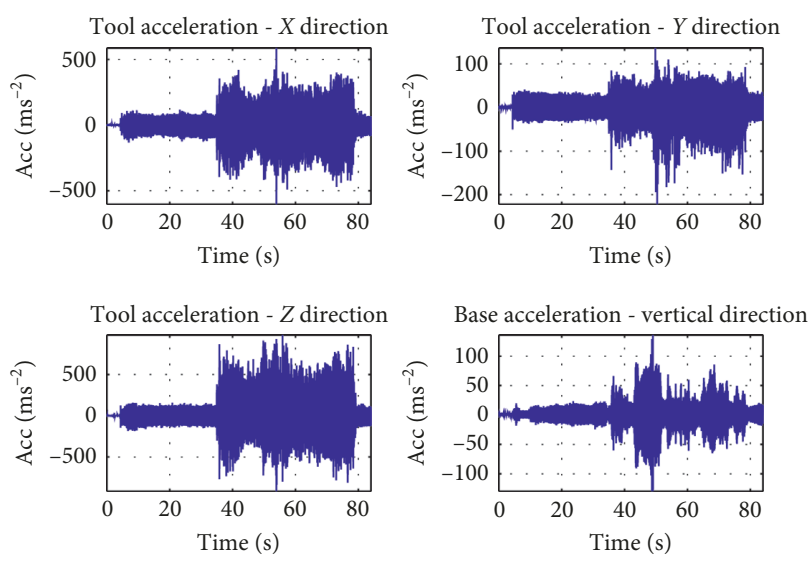

(b)

FIgURE 2: Timed evolutions of acquired acceleration for the case of squared shape (a) and circular shape (b).

magnitude peaks; (b) the transfer function between the bed frame and the milling head, with evaluation of magnitude peaks and angle sharply changes; and (c) the coherence between the bed frame and the tool accelerations, with identification of the essential frequency range for subsequent analyses. The next step on evaluating the dynamic characteristics and their changes was supposed to be a joint timefrequency analysis, based on the signals spectrograms, using STFFT, followed by peaks identification procedure and their timed tendency, during the effective working cycle. The analysis of the tendencies within the peaks diagram was performed using a qualitative procedure, based on comparison between the both milling shapes and two particular methods, which provide qualitative and quantitative measures of vibration spectrum evolution in time. First method supposed the standard deviation of previous identified peaks, both on frequency and on time variables. Second method uses the HT algorithm [15] in order to estimate the specific parameters (distance $r h o$ and angle theta from origin to a straight line) of the linear evolutions in peaks diagram.

\section{Results and Discussions}

Firstly, it has to be mentioned that the results within this paper involved only the cases of full milling process of a squared shape (named Case I) and a circular shape (named Case II). Additionally, it was presented the test case consisting only by the tool driving (rotational motion of the milling cutter), without any other equipment parts motions and milling tool interactions with the material. Actually, this last case of analysis (named third case as follows) exclusively presents the specific dynamics of the milling cutter driving system (without technological charging due to the milling process).

Taking into account the two stages in accelerations analysis, previously presented, the next paragraph contains the first step of investigations, which involves just the frequency-domain analysis methods. Thus, the diagrams in Figure 2 present the timed evolutions of the milling head and bed frame accelerations, during the whole cycle of each task (tool driving start, mobile ensemble displacement from the rest position to the start milling point, tool head vertical positioning, effective milling, tool head withdrawal, and mobile ensemble parking). Spectral composition of acquired accelerations, in terms of normalized magnitude, is depicted in Figure 3. The significance of each graph was mentioned in the diagram title.

Supposing dynamic influences on the bed frame due to the milling process, the diagrams in Figure 4 present the transfer functions (in terms of normalized magnitude and angle) between the base and the tool-holder vibration accelerations. In the same manner, the diagrams in Figure 5 depict the evolution of coherence in respect with frequency domain. As a diminishing of vibration influences in respect to the decrease in frequency was observed, the author initially assumed the $5 \mathrm{kHz}$ high limit within the spectral diagrams. Tracking the coherence evolutions (Figure 5), it was reconsidered the frequency high limit at $2 \mathrm{kHz}$ (for the second stage analyses). Diagrams in Figures 2-5 comparatively present the first and the second cases, respectively, of experimental tests. Regarding the third case of analysis, the accelerations diagrams, according to the timed evolutions, spectral composition, and transfer and coherence functions, are grouped in Figure 6.

The second step of investigations takes into account the joint time-frequency analysis of the vibration acceleration acquired during the experiments. It was adopted the STFFT algorithm in order to evaluate the continuous changes in magnitude spectrum of signals. A special procedure for finding peaks rising above a threshold (50\% of maximum magnitude in spectrogram on the whole) was used, and the results were depicted into a peaks diagram. For the three cases within this study, the spectrograms and related-to peaks diagrams are, respectively, depicted in Figures 7-9. At this point, it has to be mentioned that the joint time-frequency analysis results, in this paper, were exclusively considered for the acceleration signal acquired on the bed frame of the $\mathrm{CNC}$ machine, the justification of this aspect being explained in the discussions section. 

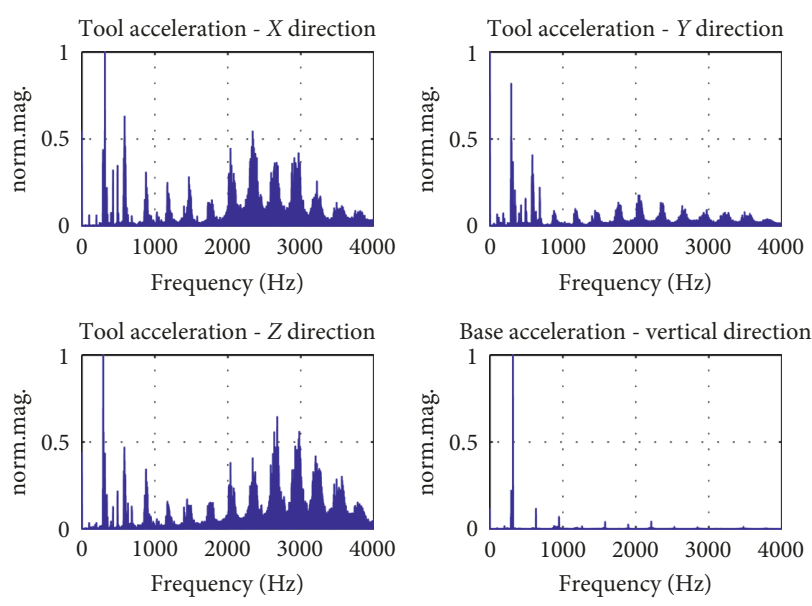

(a)
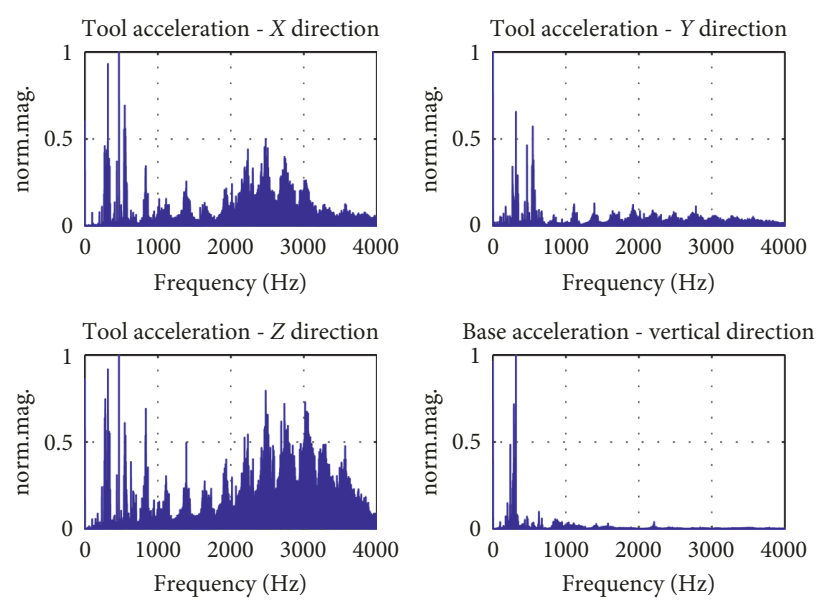

(b)

FIGURE 3: Spectral composition of acquired acceleration (normalized magnitudes) for the case of squared shape (a) and circular shape (b).
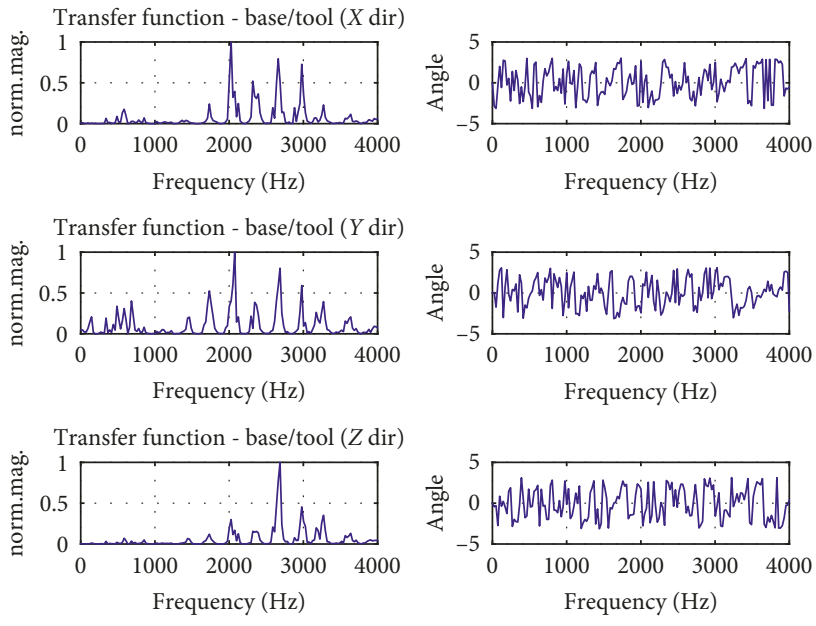

(a)
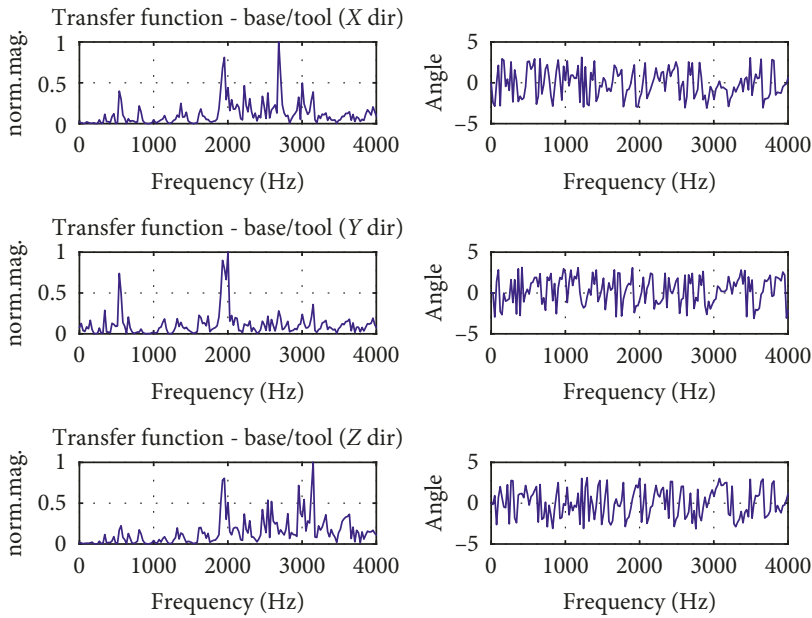

(b)

Figure 4: Transfer functions between the bed frame and milling head (normalized magnitudes on left side column and angles in radians on the right side column) for the case of squared shape (a) and circular shape (b).

Following the main purpose of the research, to identify the spectral differences between the linear and circular milling shape, the peaks diagrams were adequately cropped according to the time domain of effective milling process. Taking into account that the analysis will be mainly performed in respect with the frequency domain, the differences between the three times ranges do not imply any effect into the results. A qualitative analysis of the peaks constancy during the working process was made through the evaluation of timefrequency standard deviation for each cropped peaks diagram. The results obtained for proposed experimental cases are, respectively, presented in Figures 10(a), 11(a), and 12(a).

As it was mentioned in the previous section, a quantitative analysis of peaks diagrams was performed based on HT algorithm $[15,16]$. The classical Hough technique for curve detection is applicable if little is known about the location of a boundary, but its shape can be described as a parametric curve (e.g., straight line or conic). Its main advantages relate to that it is relatively unaffected by gaps in curves and by noise. In this study, the HT is used to extract linear features from specific datasets using the concept of parameter space. Briefly, the HT provides pertinent information regarding the lines and their length within a diagram, based on evaluation of a pair of distance and angle parameters, from an initially fixed point to each identified line. The basic output of this analysis results in a 3D diagram, wherein the sharp peaks indicate a straight line in original diagram (identified by a pair of distance and angle values into a parameter space diagram). In addition, the HT analysis was augmented with a supplementary procedure of estimation of the angle distribution, for the angular values in HT diagram corresponding to the sharp peaks (overleaping a suiting threshold). According to these aspects, the computed HT of the cropped peaks diagrams and the polar representation of the relevant angles distribution were, respectively, depicted in Figures 10(b), 11(b), and 12(b). The 

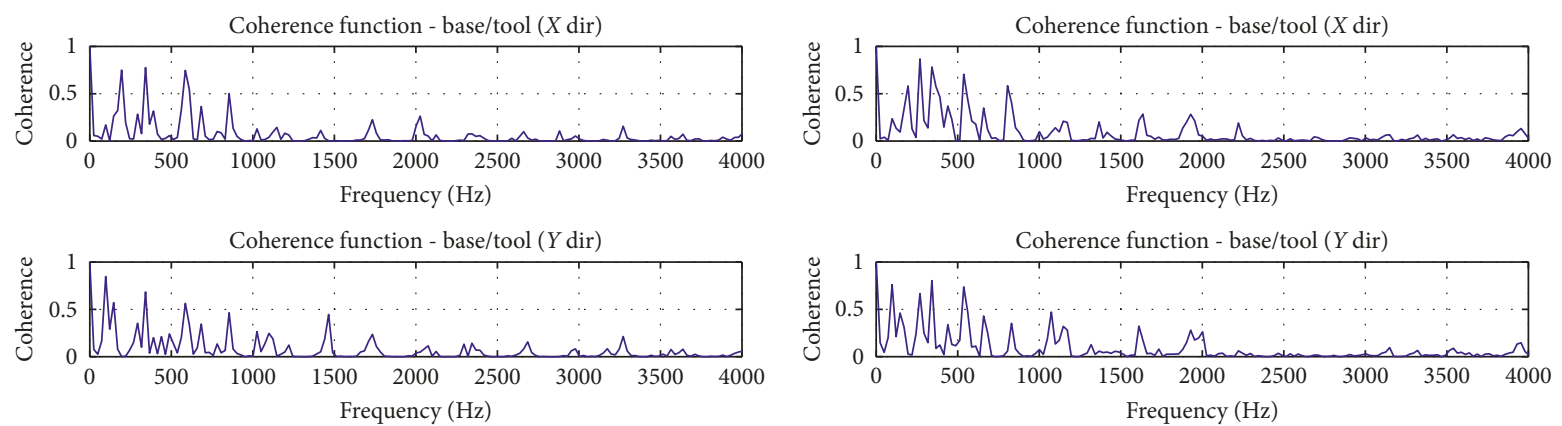

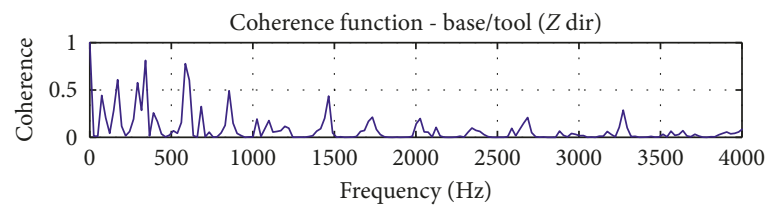

(a)

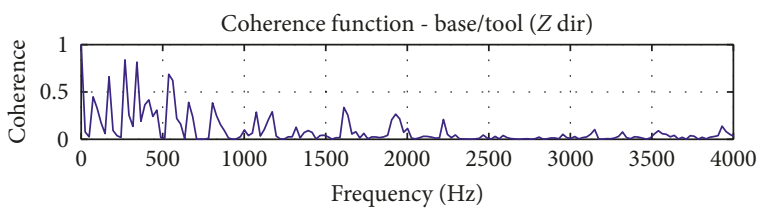

(b)

FIgURE 5: Coherence functions between the bed frame and milling head for the case of squared shape (a) and circular shape (b).
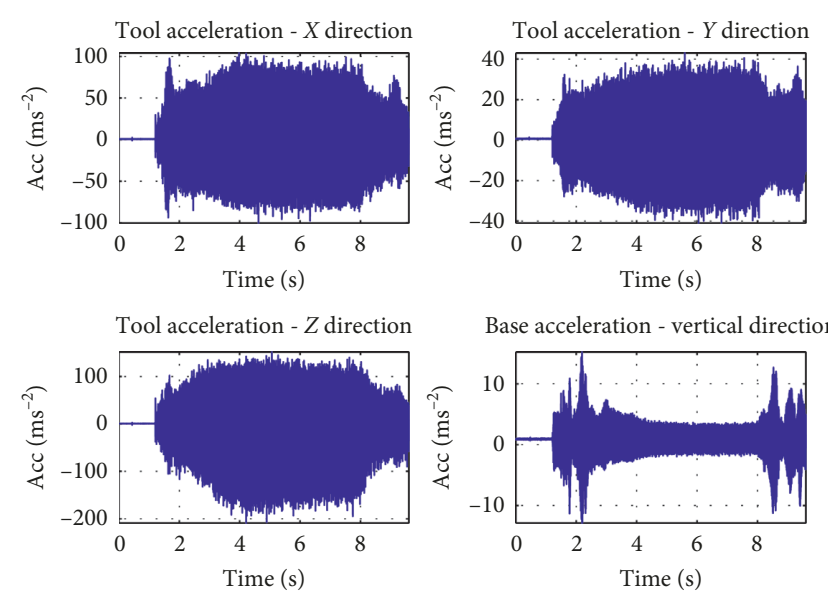

(a)
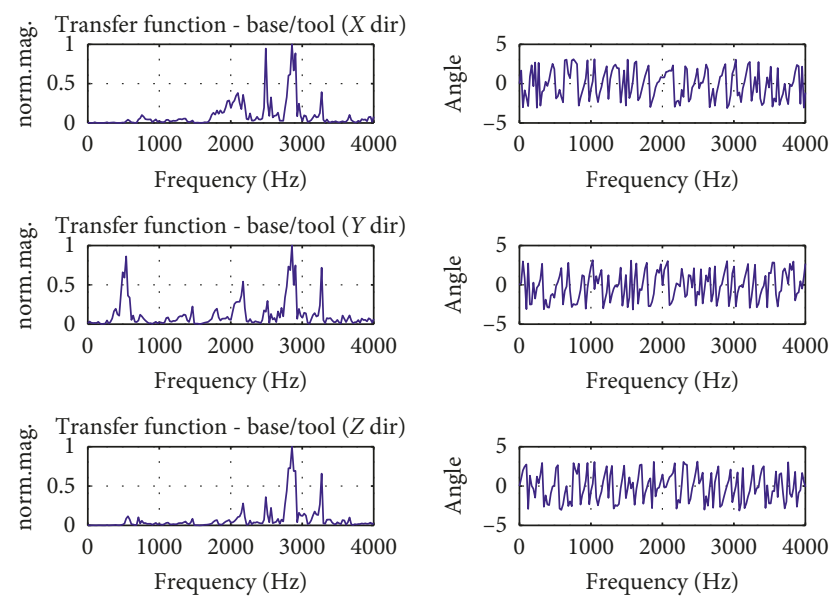

(c)
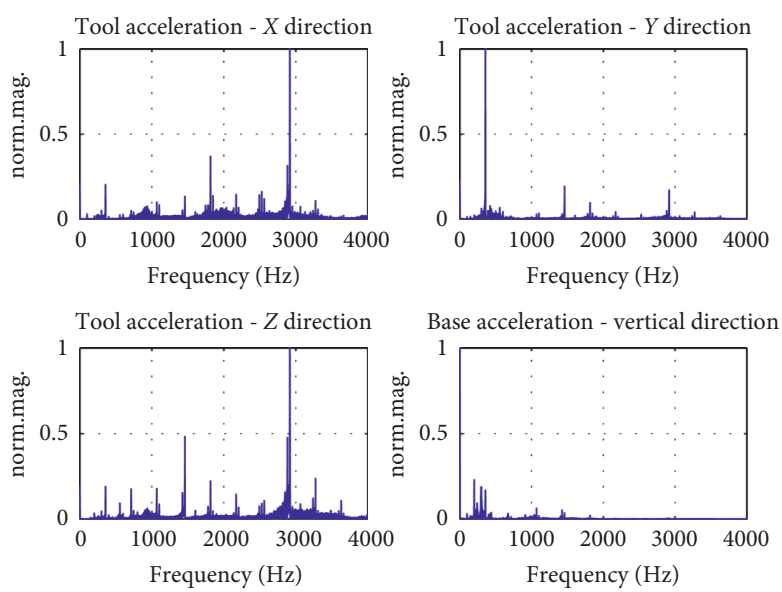

(b)
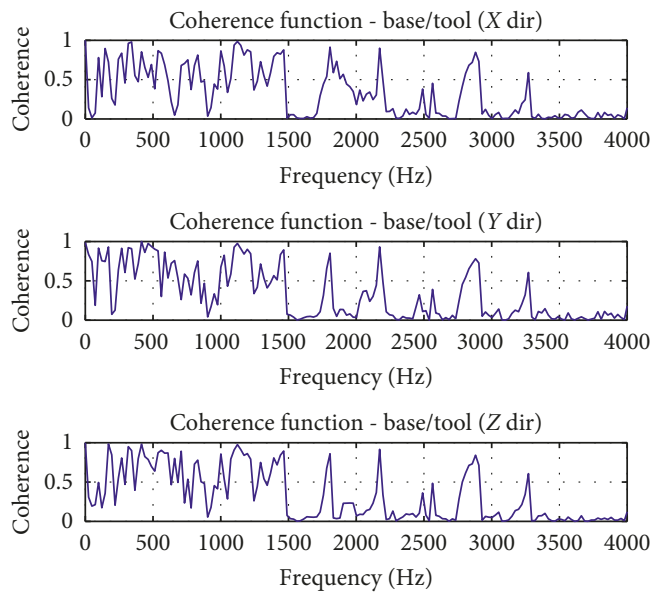

(d)

Figure 6: Timed evolution of accelerations (a), with normalized magnitudes of their spectrum (b), transfer functions (c), and coherences (d) between the bed frame and milling head, for the third case. 


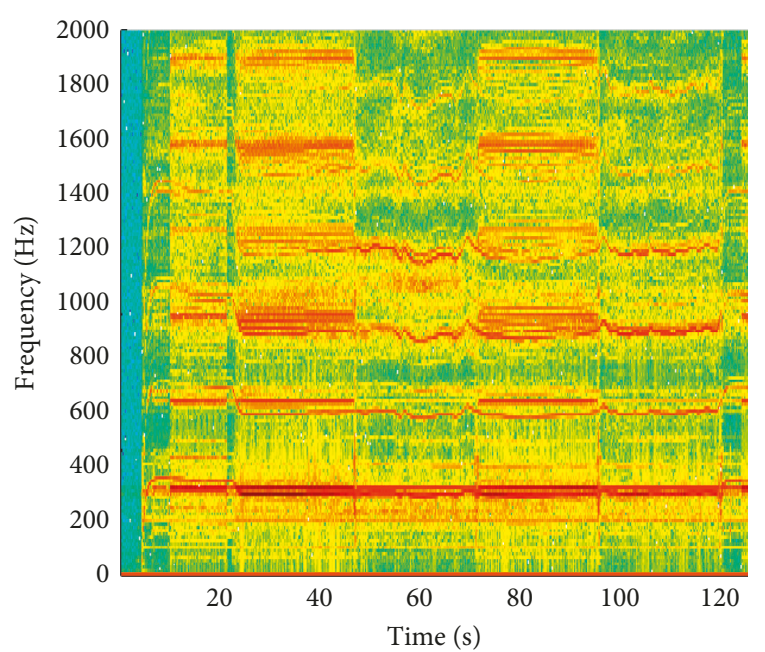

(a)

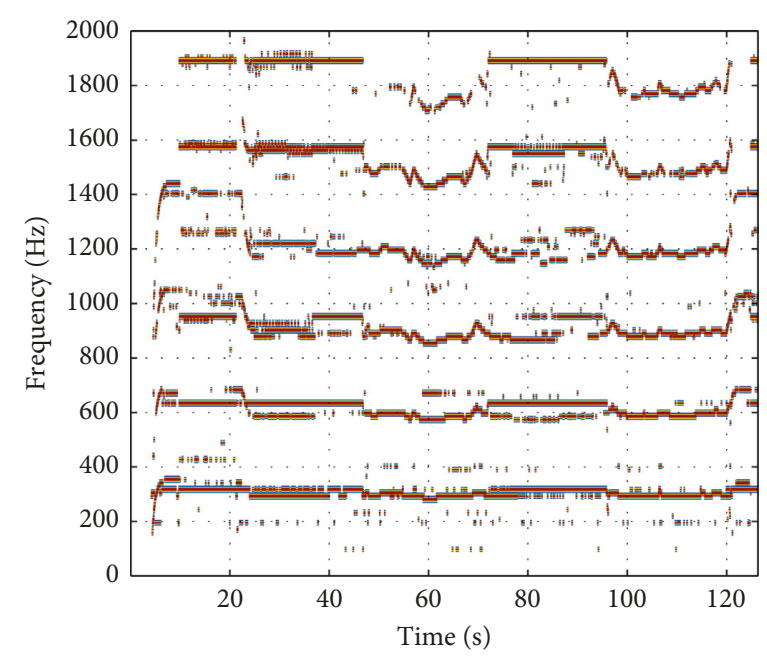

(b)

Figure 7: The spectrogram (a) with suitable peaks diagram (b) of the bed frame acceleration, for the first case of squared shape.

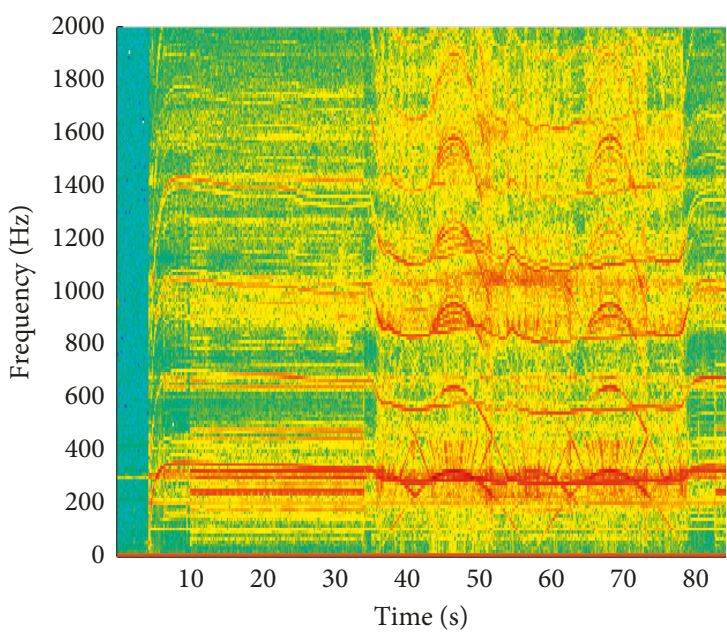

(a)

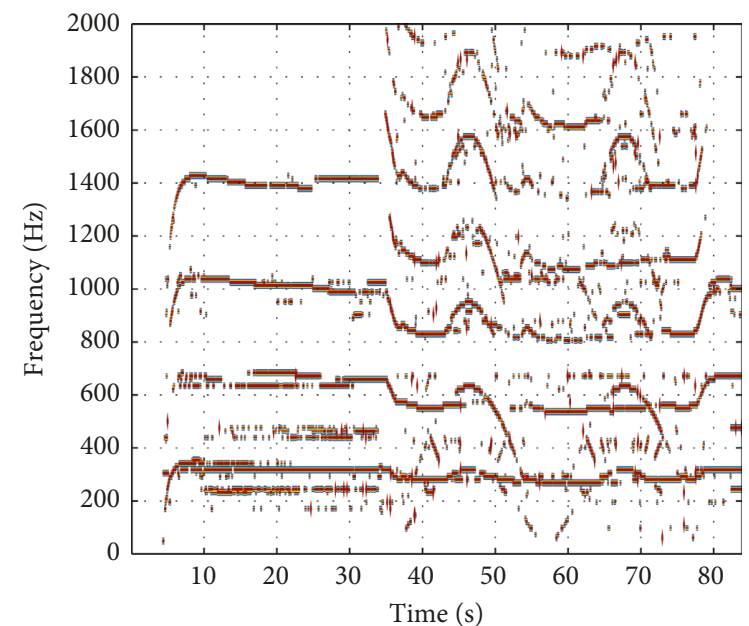

(b)

Figure 8: The spectrogram (a) with suitable peaks diagram (b) of the bed frame acceleration, for the second case of circular shape.

threshold of the peaks in HT, for histogram computation, was set at $10 \%$ of the maximum peak.

Individual and comparative analyses of the results within this study indicate a few relevant aspects as follows onto next paragraphs.

First, the magnitudes of the spectral composition (Figure 3) dignify an intensified spectrum corresponding to the second case (circular shape), independent of measuring point and direction. Taking into account the third case spectral diagrams (Figure 6(b)) results that the bed frame vibration can reveal more accurately the particularities into acceleration spectrum. Viewing the transfer functions (Figure 4), the same densification of the peaks within the magnitude diagrams can be observed. In addition, the angle changes became more sharply for the circular milling case.

As it was previously mentioned, the coherences between the bed frame and the milling head vibrations (see Figure 5) indicate that a frequency range of $(0-2000) \mathrm{Hz}$ provides relevant information regarding the machine dynamics, at least for the effective milling process analysis.

From the spectral diagrams in Figure 3, it is difficult to identify the peaks related to the particular working cycle parts. Proceeding to analyze the spectrograms in Figures 7-9, the spectral characterization of each working stage becomes evident. Going further to the peaks diagrams, respectively, in the same figures, missing of the spectral noise facilitates and improves the qualitative analysis of frequencies changes, according to the particular case and particular stage of tasks. Thus, it can be observed that fluctuations of spectral components, for square milling, are reduced, especially at low frequencies, and provide short sharply peaks during the changes of the milling regime (that indicates the moment of the changes in milling direction). In the same time, the spectrogram of the circular milling case presents a large fluctuation of frequencies during the effective milling process. 


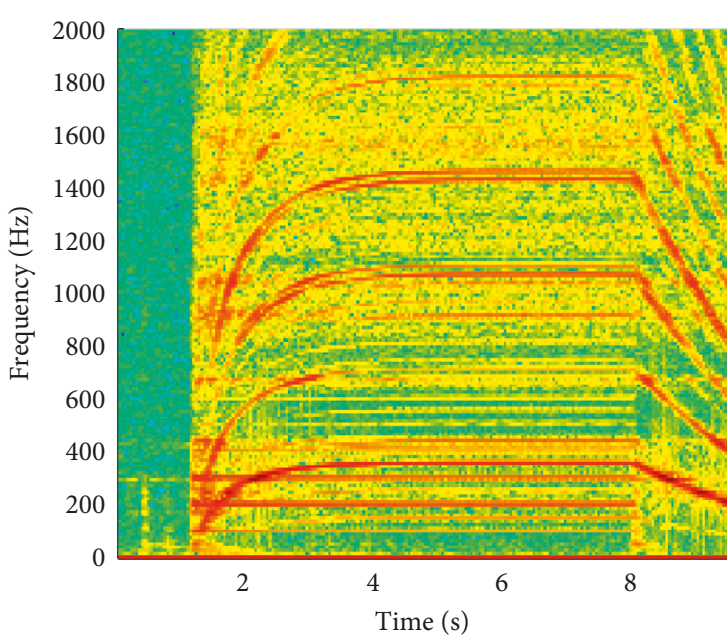

(a)

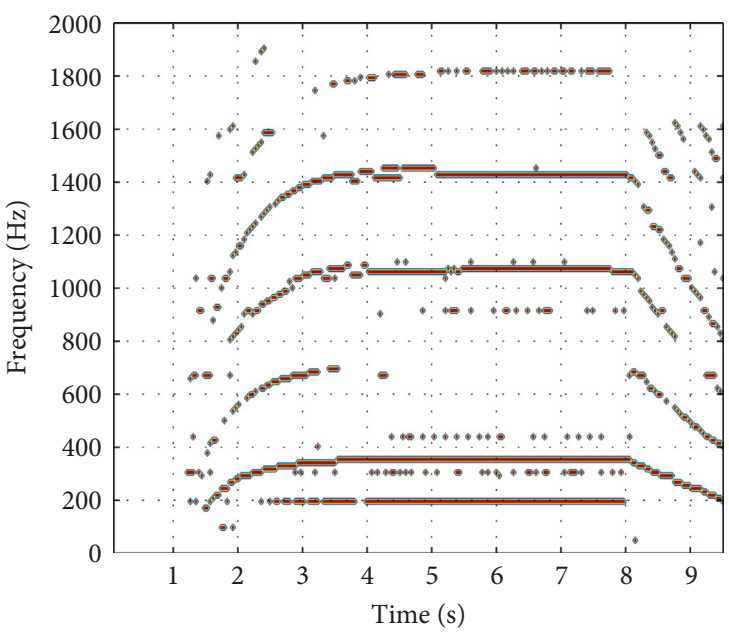

(b)

Figure 9: The spectrogram (a) with suitable peaks diagram (b) of the bed frame acceleration, for the third case.
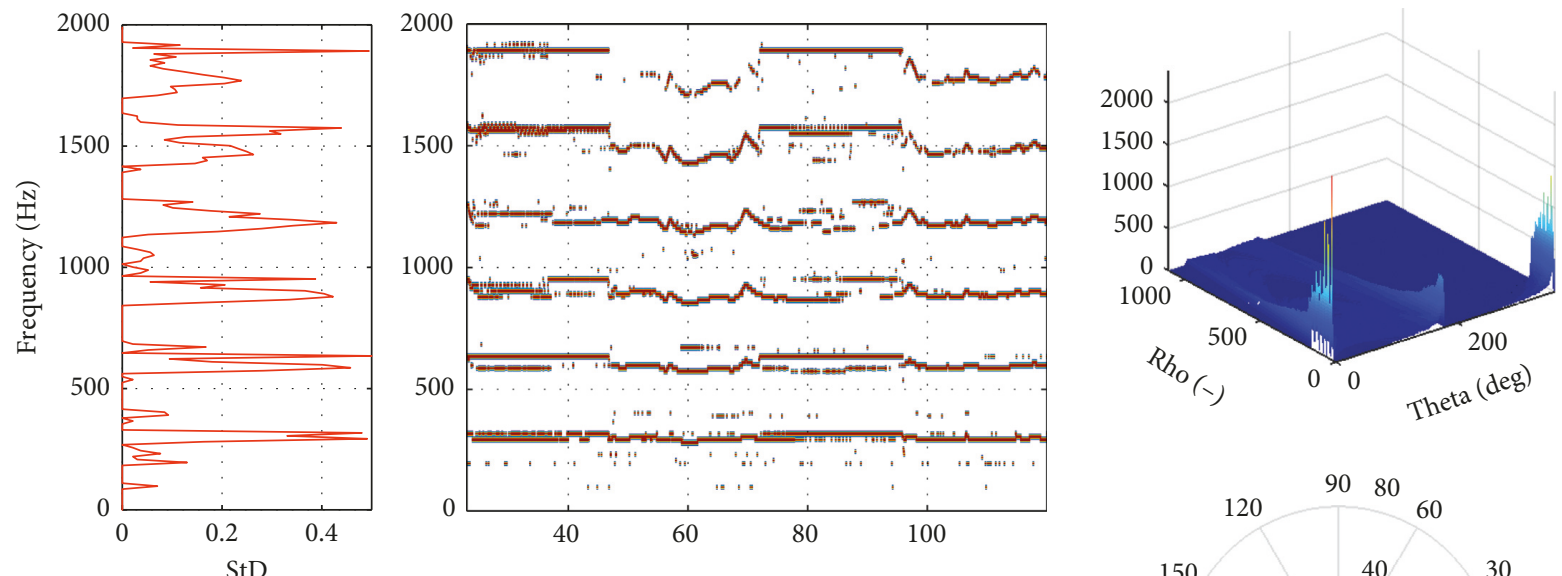

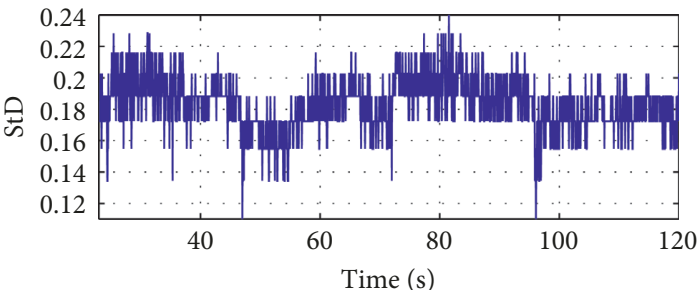

(a)

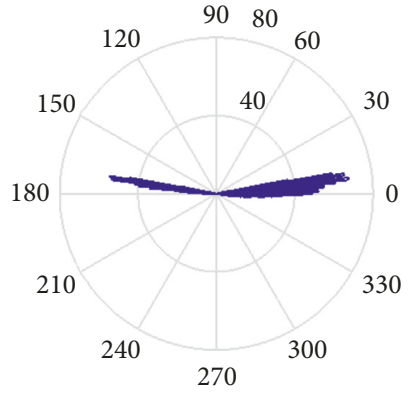

(b)

FIGURE 10: Time-frequency standard deviations (a) and HT diagram with relevant theta's polar histogram (b) of the peaks diagram (cropped within effective milling time range) of bed frame acceleration, for the first case of squared shape.

The comparative analysis of diagrams in Figures 10(a) and 11(a) reveals two types of qualitative indicators, related to the investigations that involve the time-frequency standard deviation ( $\mathrm{StD}$ ) estimators, as follows. Firstly, the specific width of the peaks within StD, in respect with frequency scale (red graphs in diagrams), grows up accordingly to the presence of circular shapes into monitored process. Secondly, the StD in respect with time scale (blue graphs in diagrams) provides a relative stable evolution, with less values, for the same case of process. Scattered aspect of the peak diagram, for the circular milling shape, comparative with the first case, leads to the previously dignified changes in StD evolutions.

The analysis of the HT diagrams (Figures 10(b) and 11(b)), together with the associate polar histograms, underlines the previous observations and provides both qualitative and quantitative tool for characterization of milling shape and of its conformity with the initial task. Even if the distribution of the angular parameter of HT, for both cases, presents two lobes (near the $0^{\circ}$ and $180^{\circ}$ ), on the second case, it can be observed that the polar distribution supplies a relative large spreading of relevant values, with approximate interval 


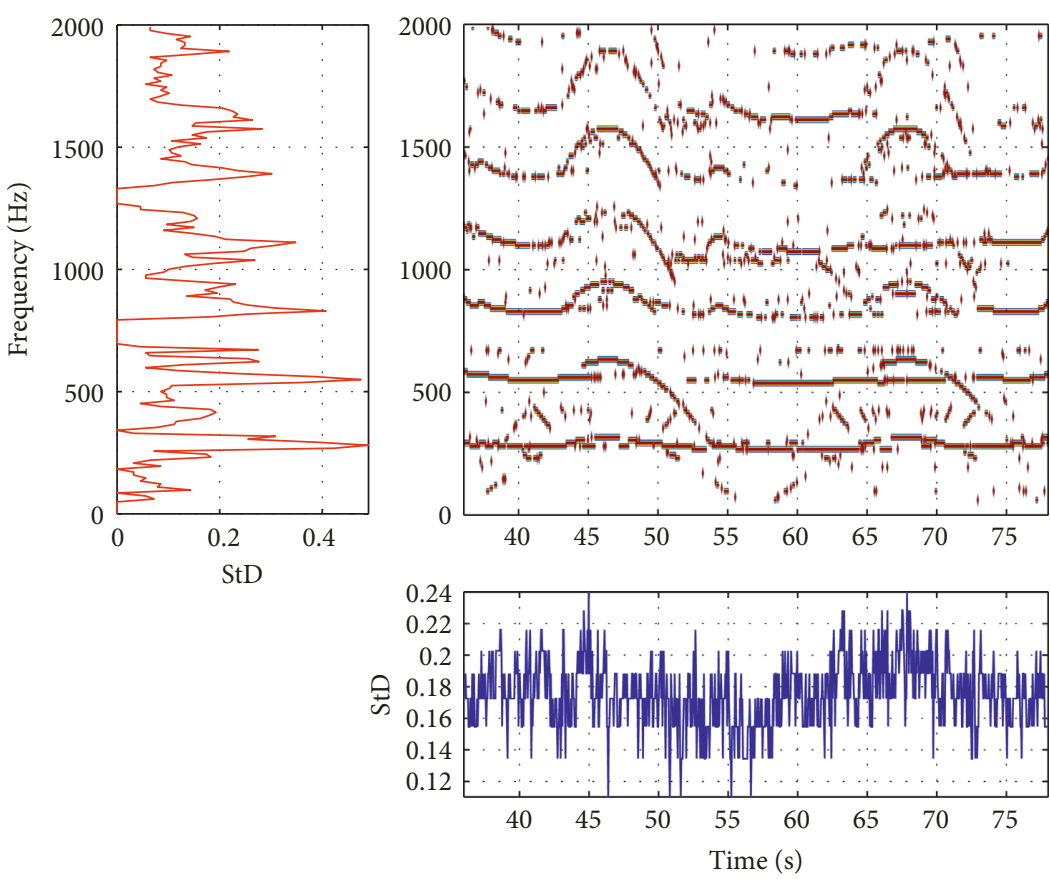

(a)
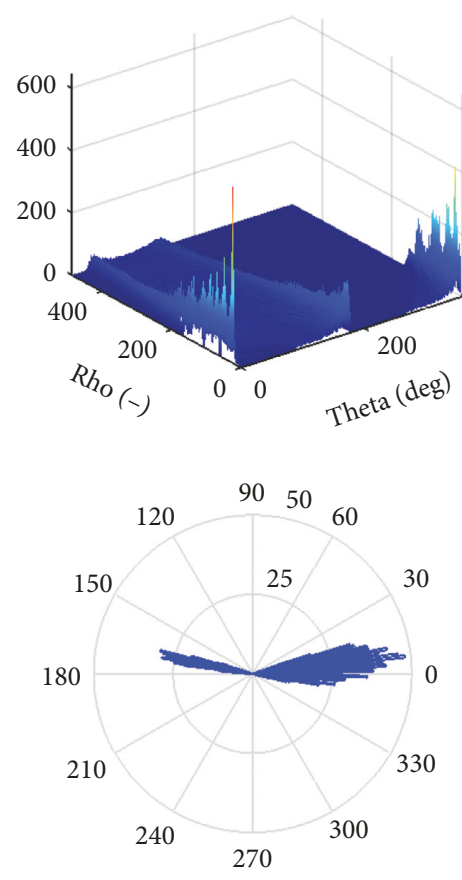

(b)

FIGURE 11: Time-frequency standard deviations (a) and HT diagram with relevant theta's polar histogram (b) of the peaks diagram (cropped within effective milling time range) of bed frame acceleration, for the second case of circular shape.
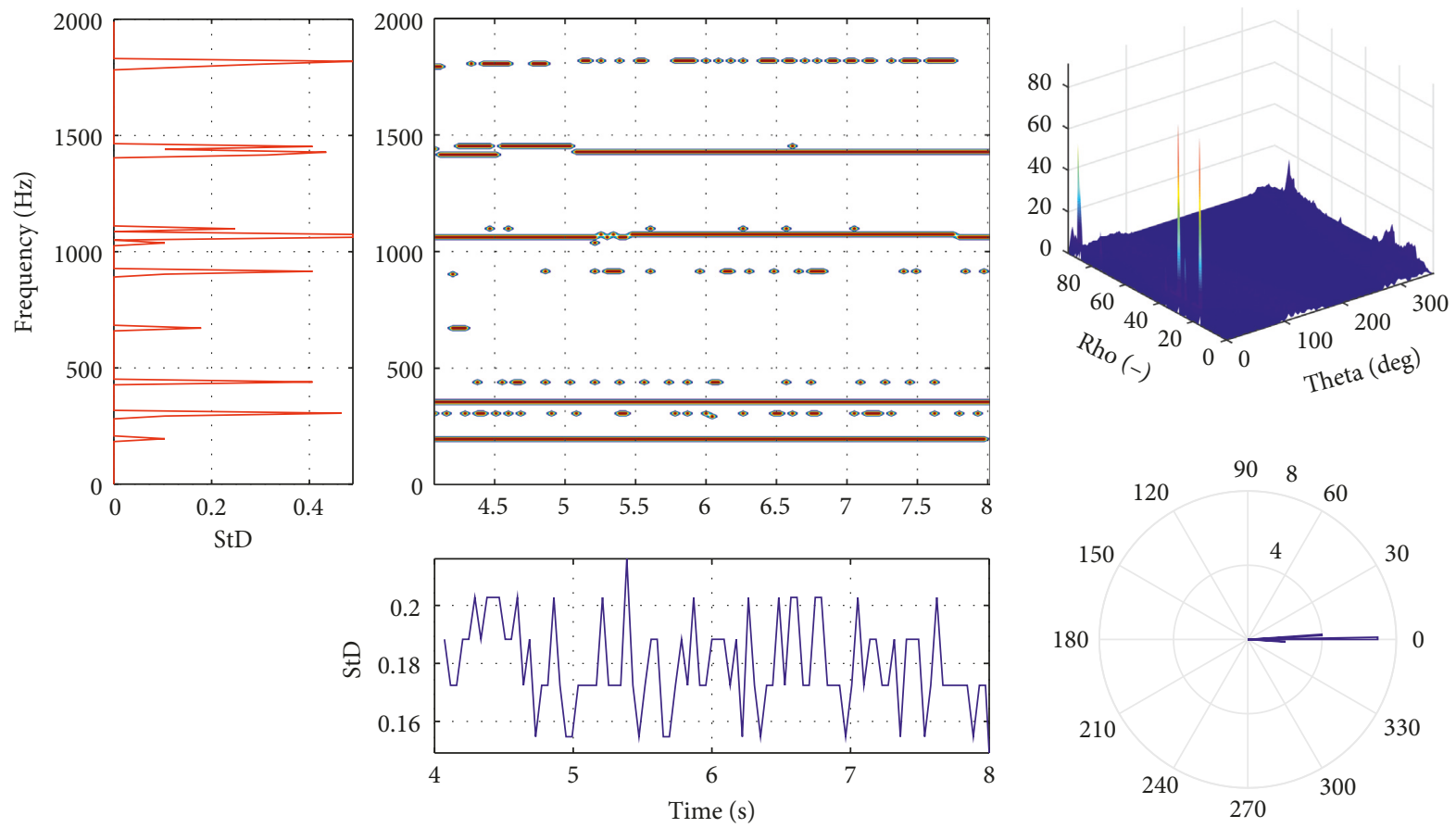

(a)

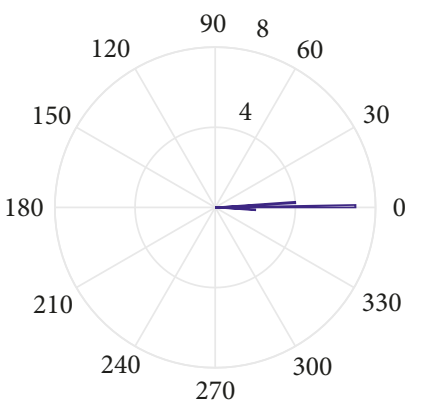

(b)

FIGURE 12: Time-frequency standard deviations (a) and HT diagram with relevant theta's polar histogram (b) of the peaks diagram (cropped within effective milling time range) of bed frame acceleration, for the third case.

width between $30^{\circ}$ and $20^{\circ}$ respectively, comparative with $10^{\circ}$ and $5^{\circ}$, for the first case. Correspondent peaks can be observed on the HT diagrams.
The results of the third case were provided in order to correlate the information regarding the exclusive tool dynamics onto the first two cases, assuming that a pertinent identification 
and analysis of milling shape conformity with initial task must involve a rejection of tool dynamics influences.

Adequate thresholds for peaks evaluation, both in spectrogram, and in HT diagram, are able to provide less error in tool trajectory estimation according to the initial imposed task.

\section{Conclusions}

This study consists of an experimental approach for solving the technical problems dealing with evaluation or estimation of the CNC machinery tool-task conformity, based on vibration measurements, and using stochastic augmented available joint time-frequency techniques. The conformity of tool trajectory, for various workpiece shapes execution, need to be identified and quantified, because the material-tool interactions have major influence over the quality of the final product (due to transitory dynamic effects that affect tool trajectory precision), finally, affecting quality and productivity of the CNC machine.

Experimental tests within this study were exclusively performed using wood workpiece samples. Theoretically speaking, the proposed analysis procedure is able to supply milling process characterization for various workpiece materials, such as metal, composites, plastics, etc. In this sense, practical implementation must take into account the specific tool-material interaction aspects, depending on material type, thus it is necessary to perform estimative studies for each particular case.

The results within this study show that the proposed computational postprocessing technique acts as a powerful analyzing tool, yielding a significant enhancement in the detection capability of milling shape accuracy and final trajectory conformity, just taking into account the vibration measurements within an operational noninvasive procedure.

This paper reports preliminary results, focused on qualitative detection performances, which appear to be very promising. The improvement in parameters estimation, intended for quantitative conformity characterization, and enlarging the workpiece material domain, will be discussed in a forthcoming work.

\section{Data Availability}

The data used to support the findings of this study are available from the corresponding author upon request.

\section{Conflicts of Interest}

The author declares that there are no conflicts of interest.

\section{References}

[1] Y. Altintas, Manufacturing Automation: Principles of Metal Cutting, Machine Tool Vibrations and CNC Design, Cambridge University Press, Cambridge, UK, 2000.

[2] A. G. Rehorn, J. Jiang, and P. E. Orban, "State-of-the-art methods and results in tool condition monitoring: a review," International Journal of Advanced Manufacturing Technology, vol. 26, pp. 693-710, 2005.
[3] C. O. Izelu, S. C. Eze, B. U. Oreko et al., "Response surface methodology in the study of induced machining vibration and work surface roughness in the turning of $41 \mathrm{Cr} 4$ alloy steel," International Journal of Emerging Technology and Advanced Engineering, vol. 3, pp. 13-17, 2013.

[4] S. K. Choudhury, N. N. Goudimenko, and V. A. Kudinov, "On-Line control of machine tool vibration in turning," International Journal of Machine Tools and Manufacture, vol. 37, pp. 801-811, 1997.

[5] D. E. Dimla, "The correlation of vibration signal features to cutting tool wear in a metal turning operation," International Journal of Advanced Manufacturing Technology, vol. 19, no. 10, pp. 705-713, 2002.

[6] D. Pop, L. Morar, E. Câmpean et al., "Experimental modal analysis of a milling machine spindle-tool holder-cutter assembly," Acta Technica Napocensis, Series: Applied Mathematics and Mechanics, vol. 55, no. 1, pp. 233-238, 2012.

[7] M. H. N. Widiyarto, D. G. Ford, and C. Pislaru, "Evaluating the structural dynamics of a vertical milling machine," Transactions on Engineering Sciences, vol. 44, pp. 421-430, 2003.

[8] K. Patoommakesorna, F. Vignata, and F. Villeneuve, "A new Straight Line Matching Technique by Integration of Visionbased image processing," Procedia CIRP, vol. 41, pp. 777-782, 2016.

[9] L. Fernandez-Robles, G. A. E. Alegre, and N. Petkov, "Cutting edge localisation in an edge profile milling head," in Proceedings of Part II, 16th International Conference on Computer Analysis of Images and Patterns (CAIP 2015), vol. 9257, pp. 336-347, Springer-Verlag New York, Inc., New York, NY, USA, September 2015.

[10] Y. Wu, D. Zhao, S. Jing et al., "Dynamic testing and fault diagnosis of CNC based on the method of cepstrum identification," International Journal of Control and Automation, vol. 7, no. 8, pp. 209-220, 2014

[11] I. Zaghbany and V. Songmene, "Estimation of machine-tool dynamic parameters during machining operation through operational modal analysis," International Journal of Machine Tools and Manufacture, vol. 49, no. 12-13, pp. 947-957, 2009.

[12] C. F. Bisu, A. Gérard, M. Zapciu et al., "The milling process monitoring using 3D envelope method," Journal of Advanced Materials Research, vol. 423, pp. 77-88, 2012.

[13] C. Debeleac, "Non-linear approaches on dynamics of multibody mechanical systems with advanced computing tools," in Proceedings of the 10th WSEAS international conference on Automation \& information ICAI'09, Prague, Czech Republic, 2009, ISBN 978-960-6766-83-1, ISSN 1790-2769.

[14] L. Gelman, "The new second and higher order spectral technique for damage monitoring of structures and machinery," International Journal of Prognostics and Health Management, vol. 7, 2014 ISSN 2153-2648.

[15] K. Glossop, P. L. G. Lisboa, and P. C. Russel, "An implementation of the Hough transformation for the identification and labeling of fixed period sinusoidal curves," Computer Vision and Image Understanding, vol. 74, no. 1, pp. 96-100, 1999.

[16] C. F. Olson, "Constrained Hough transforms for curve detection," Computer Vision and Image Understanding, vol. 73, no. 3, pp. 329-345, 1999. 


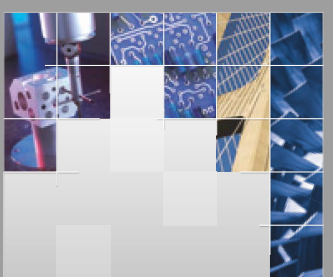

\section{Enfincering}
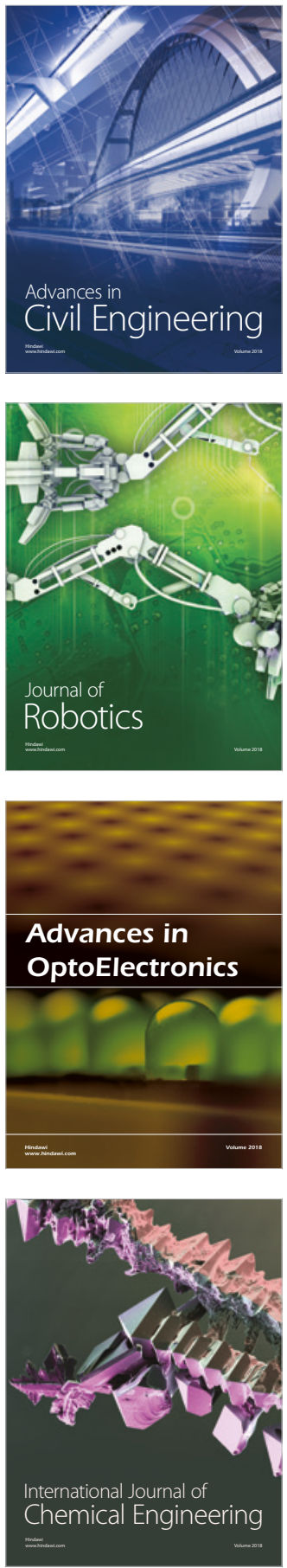

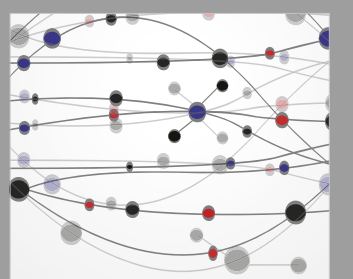

\section{Rotating \\ Machinery}

The Scientific World Journal

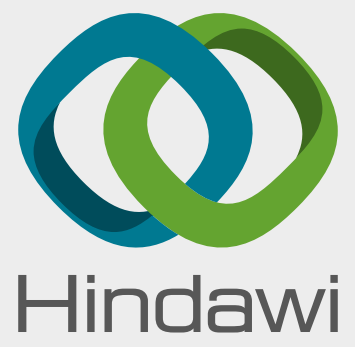

Submit your manuscripts at

www.hindawi.com
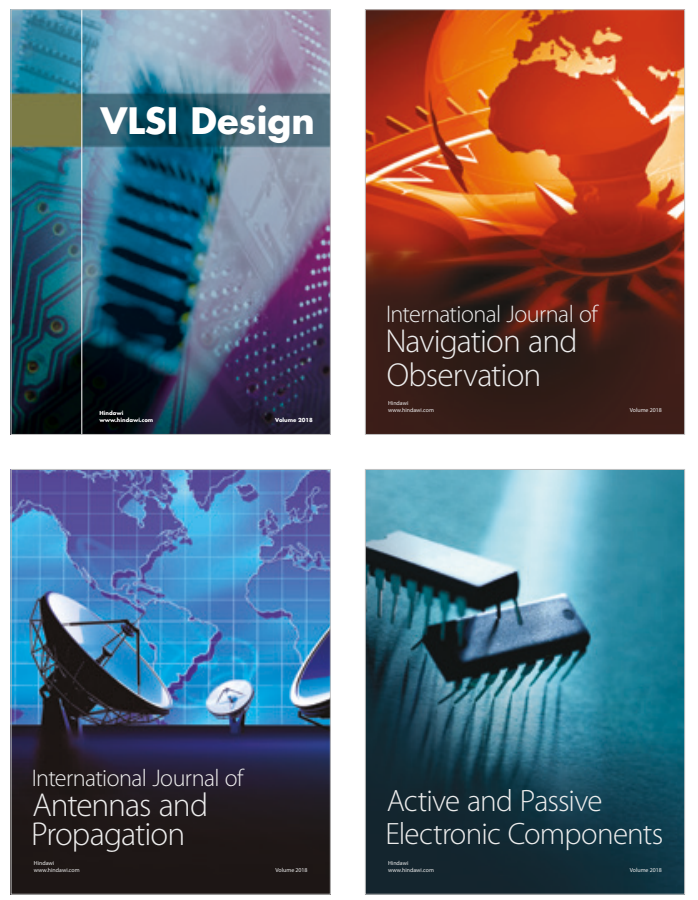
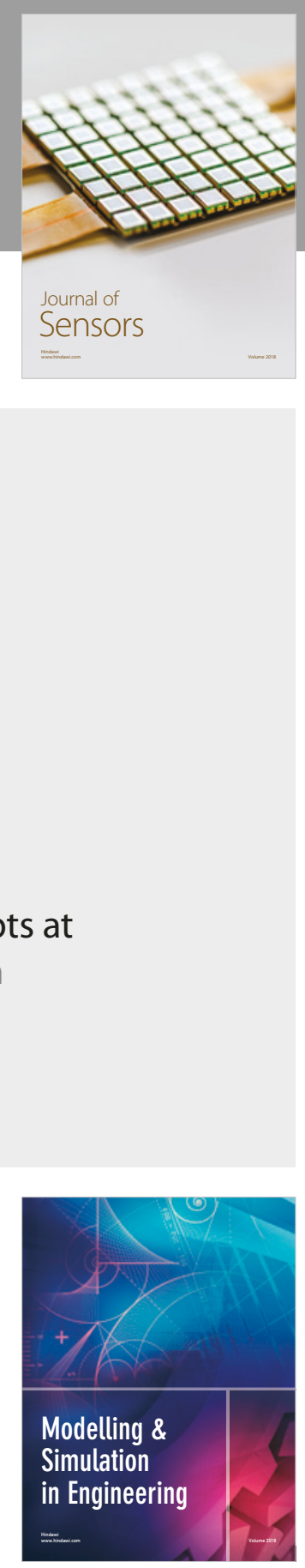

\section{Advances \\ Multimedia}
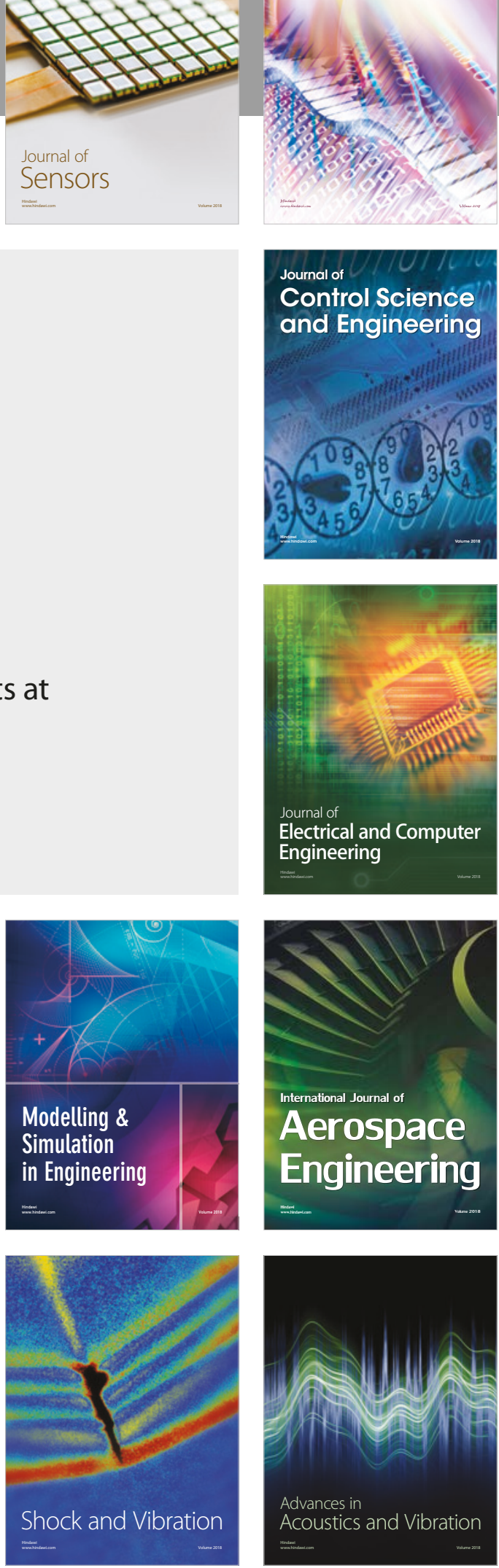